\title{
Medulloblastoma with soft-tissue and skeletal metastases in an adult: A case report
}

\author{
DIANZHONG GENG ${ }^{1}$, XIAOHUA SONG ${ }^{2}$, JING LIU $^{3}$, ZESHUN YU $^{1}$ and FANGLING NING ${ }^{1}$ \\ ${ }^{1}$ Department of Oncology, Binzhou Medical University Hospital, Binzhou, Shandong 256603; \\ Departments of ${ }^{2}$ Obstetrics and Gynecology and ${ }^{3}$ Regular Physical Examination Centre, \\ Binzhou People's Hospital, Binzhou, Shandong 256610, P.R. China
}

Received November 9, 2014; Accepted June 3, 2015

DOI: $10.3892 / \mathrm{ol} .2015 .3595$

\begin{abstract}
Medulloblastoma (MB) is a highly malignant primary brain tumor, which occurs in the cerebellum or posterior cranial fossa. MB is most commonly identified in children $<10$ years of age. The disease is rare in adults, affecting patients aged between 30 and 50 years of age, with an incidence of 0.5 cases per 1,000,000 individuals. Extraneural metastases are reported in $7-10 \%$ of cases, most commonly involving the bones and more rarely involving the lymph nodes, visceral organs and bone marrow. The current study presents the case of a 36-year-old male who underwent a gross total resection followed by radiation therapy to the craniospinal axis for the treatment of MB. The patient subsequently developed widespread metastasis, which involved the soft tissue of the occipital bone. Subsequently, the patient was administered palliative radiotherapy and initially exhibited a good clinical response. However, the patient succumbed at 18 months post-diagnosis due to dissemination of the disease. The literature on the extraneural metastasis of MB is also reviewed in the current study.
\end{abstract}

\section{Introduction}

Medulloblastoma (MB) is the most common primary brain tumor in children; however, the malignancy rarely occurs in adults (1). In total, 7-10\% of patients diagnosed with MB develop extraneural metastases, which most commonly involve the bone and lymph nodes, and recently, the frequency of reported extraneural metastases has increased (2-4). The visceral organs are less common sites of extraneural spread in medulloblastoma (5). It is generally believed that the overwhelming majority of cases reported with extraneural

Correspondence to: Dr Fangling Ning, Department of Oncology, Binzhou Medical University Hospital, 661 Huanghe 2nd Road, Binzhou, Shandong 256603, P.R. China

E-mail: ningfangling@126.com

Key words: extraneural, medulloblastoma, skeletal metastases, adult metastasis occur in patients who underwent a craniotomy (6). At present, typical treatments for high-risk childhood MB include maximal surgery, craniospinal radiation therapy and adjuvant chemotherapy (7-9). The 2- and 5-year overall survival rates following diagnosis of extraneural metastasis have been reported to be 31 and $26 \%$, respectively (10). In general, the survival rate is considered to be poor. In the current study, the case of a 36-year-old male MB patient with recurrent disease involving the soft tissues of the occipital bone is presented, as well as the subsequent treatment course of the patient. In addition, the extraneural metastasis of MB is discussed. Written informed consent was obtained from the patient for the publication of this case report and any accompanying images.

\section{Case report}

On November 15, 2009, a 36-year-old male presented at the Binzhou Medical University Hospital (Binzhou, China) with nausea and vomiting that had persisted for 5 days, but was not accompanied by headache, visual impairment or ataxia. The patient had no significant previous medical history. Cranial magnetic resonance imaging (MRI) revealed the presence of a lesion in the fourth ventricle. Total spinal MRI revealed no evidence of additional central nervous system disease. Subsequently, three days after presentation, the patient underwent a posterior fossa craniotomy with gross total resection of the tumor, in which the cerebrospinal fluid (CSF) circulation was restored, without the insertion of a drainage strip. Following histopathological analysis, the resected tumor was diagnosed as average risk MB (Fig. 1A). The patient was administered external beam radiation therapy to the craniospinal axis at a dose of 36 Gy for 4 weeks, followed by a boost to the posterior fossa at a dose of $54 \mathrm{~Gy}$ for 6 weeks, completing therapy in early February 2010. Simultaneously, temozolomide (100 mg/day) was administered orally $1 \mathrm{~h}$ before each radiotherapy treatment up to the end of radiotherapy.

One month after the completion of radiotherapy, the patient noticed an occipital mass without clinical symptoms, but did not seek medical attention. The tumor grew to $\sim 4 \times 5 \times 6 \mathrm{~cm}^{3}$ in size, until September 28, 2010, when a surgical excision was performed. The histopathological diagnosis was also of MB (Fig. 1B). The patient experienced left-sided chest pain, and bone and chest computed tomography (CT) scans revealed 

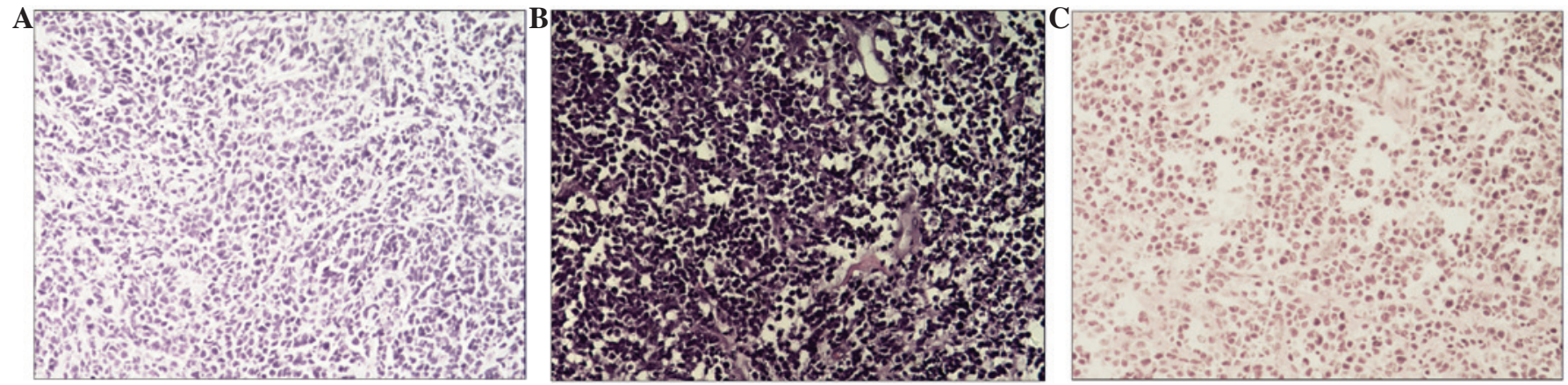

Figure 1. Histopathological staining of the (A) craniocerebral tumor, (B) occipital tumor and (C) fourth left rib metastases revealing tumors composed of a high density of cells, with unclear cytoplasm and round or oval-shaped nuclei with increased euchromatin (hematoxylin and eosin staining; magnification, x200).

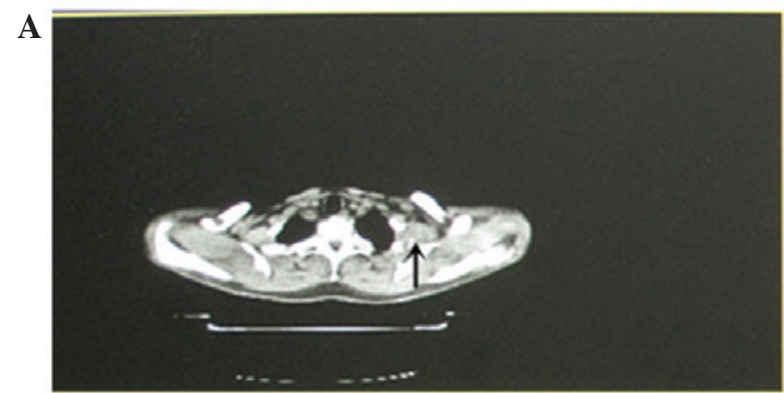

C
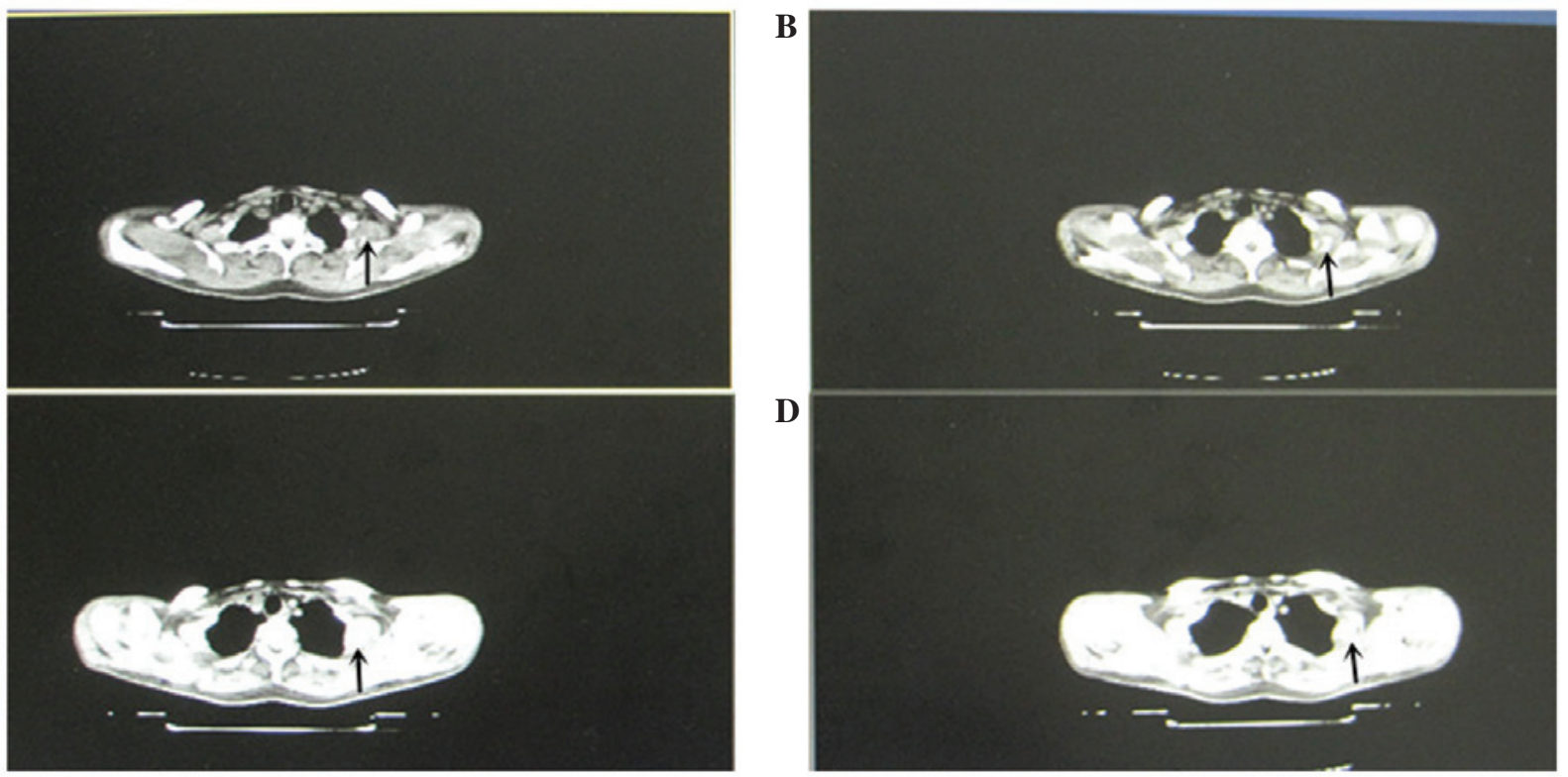

Figure 2. Axial chest computed tomography scan of the thoracic inlet revealing bone destruction and local soft tissue mass formation in the fourth rib. (A) Bone destruction at the upper edge of the fourth rib, with a small area of local soft tissue mass formation at the thoracic inlet. (B) Evident bone destruction and local soft tissue mass formation in the fourth rib. (C) Bone destruction in the fourth rib, with a large area of local soft tissue mass formation. (D) Local soft tissue mass formation at the lower edge of the fourth rib.

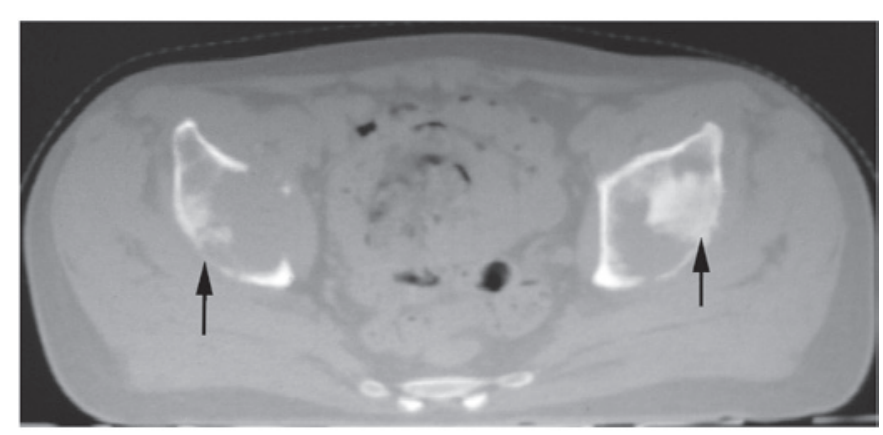

Figure 3. Pelvic computed tomography demonstrating multiple bone metastases (arrows)

fourth left rib metastasis (Fig. 2). On December 16, 2010, the fourth left rib was resected. Histopathological analysis revealed metastatic small cell cancer in the bone and soft tissue, which was associated with the clinical response. Subsequently, according to the histopathological analysis of the fourth left rib resection and considering the previous biopsy, metastatic MB was diagnosed (Fig. 1C). One month after surgery, the patient experienced pain in multiple locations. Repeat bone and CT scans revealed multiple bone metastases in several ribs, the pelvis (Fig. 3), the humeri and the femora. Consequently, the patient was administered palliative radiotherapy and oxycodone hydrochloride prolonged-release tablets $(20 \mathrm{mg} / 12 \mathrm{~h})$ to relieve the symptoms. The patient succumbed at 18 months post-diagnosis due to dissemination of the disease.

\section{Discussion}

MB is the most common primary brain tumor in children, however, the occurrence of MB in adults is extremely rare, with an incidence of 0.5 cases per 1,000,000 individuals. In adults, $80 \%$ of MB cases occur prior to the end of the fourth decade of life. Extraneural metastasis in MB is rare, occurring in 7-10\% of MB patients, and $1-2 \%$ of patients at the time of the initial diagnosis (10). At present, treatment for high-risk childhood MB includes maximal surgery, craniospinal radiation therapy 
and adjuvant chemotherapy. Surgical resection is the standard primary treatment for MB (10). Surgical techniques have three primary goals: i) Restoring normal CSF flow; ii) maximal tumor resection; and iii) obtaining tissue for histopathology. Radiotherapy to the craniospinal axis at a dose of 36 Gy for 4 weeks followed by a boost to the posterior fossa at a dose of 54 Gy for 6 weeks has been adopted as standard practice. In a French multicenter study, a radiotherapy dose of $<50$ Gy to the posterior fossa was found to be a poor prognostic factor for MB patients (11). The extent of resection and the interval between surgery and radiotherapy were also prognostic factors of MB $(12,13)$. Chemotherapy has been administered for the treatment of $\mathrm{MB}$ for $>20$ years; initially, the regimens were the same as those used to treat childhood MB, however, temozolomide has begun to replace older chemotherapeutic combinations with a reasonable efficacy and decreased adverse effects $(7,14)$.

The clinical presentation and imaging features of the disease are different in adults compared with children with MB, and have the following characteristics: i) The craniosynostosis of adults has closed and thus the compensatory ability of the cranial space is poor. The cranial sutures of infants and small children have not closed, and thus the effects of intracranial pressure (ICP) differ. In infants, the fontanels bulge when the ICP is too high. However, the course of the disease in adults is relatively short. In the present case, the patient presented with nausea and vomiting that had persisted for 5 days. ii) More than half of adult MBs are located in the cerebellar hemispheres, whereas MBs in children are usually located in the cerebellar vermis; cystic necrosis and calcification within the tumor are more common in adults with MB than in children (8). iii) The brain and spinal cord of adults are mature and thus have good tolerance for post-operative radiotherapy.

Infiltration of the subarachnoid space and consequent metastatic spread via the CSF is common in MB (10), but extraneural metastasis is extremely rare. The rarity of extraneural metastasis may be due to the following factors: i) The lack of intraparenchymal lymphatic vessels; ii) the collapse of tumor-adjacent intracerebral veins; iii) the inability of the intracerebral environment for the selection of metastatic malignant clones; iv) the presence of the blood-brain barrier; and v) the short survival time of the affected patients. Notably, the majority of patients with extraneural metastasis have undergone craniotomy. In a previous study, Pasquier et al (6) reported that 237/247 (96.0\%) of patients with extraneural metastasis had previously undergone craniotomy. Patients with unresectable tumors exhibit a worse outcome, often succumbing to primary disease prior to the development of extraneural metastasis. Extraneural metastases may be induced iatrogenically, such as peritoneal metastasis with ventriculoperitoneal shunts (15), hematogenous metastasis secondary to disruption of blood vessels during surgery, seeding on the incision and direct extensions from the central nervous system (16).

In the present case, we hypothesize that the dural suture may not have been sufficiently tight intraoperatively and thus, implantation metastasis occurred around the incision following the procedure. Radiotherapy was administered to the general target area; the surgical incision was not provided with targeted attention, and additionally, as this was the head and neck junction, coldspot (insufficient radiation dose deposition) was prone to emerge in the succession of the radiation field, which leads to the deficiency of the radiotherapy dose. The clinical course of osseous metastasis of this patient was possibly initial transfer of the tumor cells to the neck and then into the venous system, followed by transfer to multiple bone tissues. For a patient to be classified as intermediate risk according to the MB classification (17), further study is required to ascertain whether the application of preventive chemotherapy is required and when to administer radiotherapy, or whether it should be implemented concurrently (17). Thus, to prevent metastasis, during surgery the surrounding tissue must be protected to reduce damage, the surgical field must be kept clean, and the post-operative suture must be closed or the dura repaired, ensuring enhancement of the 'tumor-free concept'. If signs of metastasis are identified, prophylactic radiotherapy of the whole brain and spinal cord should be administered promptly following surgery, close attention should be paid to the surgical incision to avoid coldspot, the radiation dose should be increased and chemotherapy should be implemented.

When the current patient with MB developed an extraneural metastasis, the disease progressed rapidly. After the termination of active treatment, the patient succumbed to the disease 18 months after diagnosis. The outcome is thus poor in patients with MB with extraneural metastasis. In conclusion, we suggest that for a prolonged survival time in adults with $\mathrm{MB}$, greater emphasis must be placed on the possibility of systemic involvement. Increased understanding with regard to the pathogenesis of systemic metastases may be useful for the development of more aggressive multimodal therapies in the future.

\section{References}

1. Smoll NR and Drummond KJ: The incidence of medulloblastomas and primitive neurectodermal tumours in adults and children. J Clin Neurosci 19: 1541-1544, 2012.

2. Paulino AC: Long-term survival in a child with extraneural metastasis from medulloblastoma treated with chemo-radiotherapy. Med Pediatr Oncol 40: 396-397, 2003.

3. Clement J, Varlotto J, Rybka W, Frauenhoffer E and Drabick JJ: Unusual case of recurrent extraneural metastatic medulloblastoma in a young adult: Durable complete remission with Ewing sarcoma chemotherapy regimen and consolidation with autologous bone marrow transplantation and local radiation. $\mathrm{J}$ Clin Oncol 31: e316-e319, 2013.

4. Wendland MM, Shrieve DC, Watson GA, Chin SS and Blumenthal DT: Extraneural metastatic medulloblastoma in an adult. J Neurooncol 78: 191-196, 2006.

5. Koschny R, Ahnert P and Holland H: Medulloblastoma: Therapy with bortezomib/tumor necrosis factor-related apoptosis-inducing ligand. In: Tumors of the Central Nervous System. Vol 8. Hayat MA (ed). Springer, New York, NY, pp77-84, 2012.

6. Pasquier B, Pasquier D, N'Golet A, Panh MH and Couderc P: Extraneural metastases of astrocytomas and glioblastomas: Clinicopathological study of two cases and review of literature. Cancer 45: 112-125, 1980.

7. van den Bent MJ, Stupp R, Brandes AA and Lacombe D: Current and future trials of the EORTC brain tumor group. Onkologie 27: 246-250, 2004.

8. Koral K, Gargan L, Bowers DC, Gimi B, Timmons CF, Weprin B and Rollins NK: Imaging characteristics of atypical teratoid-rhabdoid tumor in children compared with medulloblastoma. AJR Am J Roentgenol 190: 809-814, 2008.

9. Zhang N, Ouyang T, Kang H, Long W, Thomas B and Zhu S: Adult medulloblastoma: Clinical characters, prognostic factors, outcomes and patterns of relapse. J Neurooncol. May 31, 2015 (Epub ahead of print). 
10. Caracciolo V and Giordano A: Medulloblastoma: Classification (A Review). In: Tumors of the Central Nervous System. Hayat MA (ed). Volume 8. Springer, New York, NY,pp23-86, 2012.

11. Padovani L, Sunyach MP, Perol D, Mercier C, Alapetite C, Haie-Meder C, Hoffstetter S, Muracciole X, Kerr C, Wagner JP, et al: Common strategy for adult and pediatric medulloblastoma: A multicenter series of 253 adults. Int J Radiat Oncol Biol Phys 68 : 433-440, 2007.

12. Abacioglu U, Uzel O, Sengoz M, Turkan S and Ober A: Medulloblastoma in adults: Treatment results and prognostic factors. Int J Radiat Oncol Biol Phys 54: 855-860, 2002.

13. Lai R: Survival of patients with adult medulloblastoma: A population-based study. Cancer 112: 1568-1574, 2008.

14. Hongeng S, Visudtibhan A, Dhanachai M, Laothamatus J and Chiamchanya S: Treatment of leptomeningeal relapse of medulloblastoma with temozolomide. J Pediatr Hematol Oncol 24: 591-593, 2002.
15. Berry MP, Jenkin RD, Keen CW, Nair BD and Simpson WJ: Radiation treatment for medulloblastoma. A 21-year review. J Neurosurg 55: 43-51, 1981.

16. McComb JG, Davis RL, Isaacs $\mathrm{H}$ Jr and Landing $\mathrm{BH}$ Medulloblastoma presenting as neck tumors in 2 infants. Ann Neurol 7: 113-117, 1980.

17. Rutkowski S, von Bueren A, von Hoff K, Hartmann W, Shalaby T, Deinlein F, Warmuth-Metz M, Soerensen N, Emser A, Bode U, et al: Prognostic relevance of clinical and biological risk factors in childhood medulloblastoma: results of patients treated in the prospective multicenter trial HIT'91. Clin Cancer Res 13: 2651-2657, 2007. 